\title{
Forced Oscillations of Electrical Conducting Fluid Under the Influence of Applied Magnetic Field on the Porous Boundary
}

\author{
Sanjay B. Kulkarni \\ Department of First Year Engineering (Science \& Humanities), Finolex Academy of Management and Technology, Ratnagiri, India
}

Email address:

sanjay_kulkarni1@yahoo.co

\section{To cite this article:}

Sanjay B. Kulkarni. Forced Oscillations of Electrical Conducting Fluid Under the Influence of Applied Magnetic Field on the Porous Boundary. American Journal of Software Engineering and Applications. Vol. 5, No. 5, 2016, pp. 33-39. doi: 10.11648/j.ajsea.20160505.11

Received: December 5, 2016; Accepted: December 17, 2016; Published: January 13, 2017

\begin{abstract}
Exact solution of an incompressible fluid of second order type by causing forced oscillations in the liquid of finite depth bounded by a porous bottom has been obtained in this paper. The results presented are in terms of non-dimensional elastic-viscosity parameter $(\beta)$ which depends on the non-Newtonian coefficient and the frequency of excitation $(\sigma)$ of the external disturbance while considering the porosity $(\mathrm{K})$ and magnetic parameter $(\mathrm{m})$ of the medium into account. The flow parameters are found to be identical with that of Newtonian case as $\beta \rightarrow 0, m \rightarrow 0$ and $K \rightarrow \infty$. It is seen that the effect of elastico viscosity parameter, magnetic parameter and the porosity of the bounding surface has significant effect on the velocity parameter, phase parameter, skin friction and mass flow rate. Further, the nature of the paths of the fluid particles have also been obtained with reference to the elastico viscosity parameter, magnetic parameter and the porosity of the bounding surface.
\end{abstract}

Keywords: Elastico-Viscous Fluid, Magnetic Parameter Porous Media, Mas Flow Rate, Second Order Fluid

\section{Introduction}

When a conductive fluid moves through a magnetic field, an ionized gas is electrically conductive, and the fluid is influenced by the magnetic field. Natural convection and transfer of heat is of considerable interest in problems that arises in magneto hydrodynamic (MHD) especially in the technical field due to its frequent occurrence in industrial technology and geothermal applications. The applications are wide in variety of situations where the high-temperature plasmas are applicable in nuclear fusion energy conversion, liquid metal fluids, and (MHD) power generation systems. Further, in several problems related to geophysical, petroleum, chemical and biomechanical that are usually bounded by porous medium, the problem assumes greater significance. Convective boundary layer flows are often controlled by fluid suction or injection through a porous heated wall. This process can lead to enhancement of the heat transfer coefficient or cooling of the system. Due to several applications in the fields of geo physics, metallurgy, petroleum engineering, chemical engineering, composite metal engineering and heat exchanges, the problem of mass transfer and radiation effects are unsteady MHD flows. Free convective flow embedded in a porous medium with a heat generation or absorption assumes greater significant over the last two decades. Porous media has been the subject of considerable research activity in recent years because of its several important applications notably in the flow of oil through porous rock, the extraction of geothermal energy from the deep interior of the earth to the shallow layers, the evaluation of the capability of heat removal from particulate nuclear fuel debris that may result from a hypothetical accident in a nuclear reactor, the filtration of solids from liquids, flow of liquids through ion exchange beds, drug permeation through human skin, chemical reactor for economical separation or purification of mixtures and so on.

In many chemical processing industries, slurry adheres to the reactor vessels and gets consolidated. As a result of this, the chemical compounds within the reactor vessel percolates through the boundaries causing loss of production and then consuming more reaction time. In view of such technological and industrial importance wherein the heat and mass transfer takes place in the chemical industry, the problem by considering the permeability of the bounding surfaces in the reactors attracted the attention of several investigators. An 
important application is in the petroleum industry, where crude oil is tapped from natural underground reservoirs in which oil is entrapped. Since the flow behaviour of fluids in petroleum reservoir rock depends, to a large extent, on the properties of the rock, techniques that yield new or additional information on the characteristics of the rock would enhance the performance of the petroleum reservoirs. A related bio-mechanical application is the flow of fluids in the lungs, blood vessels, arteries and so on, where the fluid is bounded by two layers which are held together by a set of fairly regularly spaced tissues.

Viscous fluid flow over wavy wall had attracted the attention of relatively few researchers although the analysis of such flows finds application in different areas, such as transpiration cooling of re-entry vehicles and rocket boosters, cross hatching on ablative surfaces and film vaporization in combustion chambers etc. Especially, where the heat and mass transfer takes place in the chemical processing industry, the problem by considering the permeability of the bounding surface in the reactors assumes greater significance. Many materials such as drilling muds, clay coatings and other suspensions, certain oils and greases, polymer melts, elastomers and many emulsions have been treated as non-Newtonian fluids. Because of the difficulty to suggest a single model, which exhibits all properties of non-Newtonian fluids, they cannot be described simply as Newtonian fluids and there has been much confusion over the classification of non-Newtonian fluids. However, non-Newtonian fluids may be classified as (i) fluids for which the shear stress depends only on the rate of shear; (ii) fluids for which the relation between shear stress and shear rate depends on time; (iii) the visco-elastic fluids, which possess both elastic and viscous properties.

Because of the great diversity in the physical structure of non-Newtonian fluids, it is not possible to recommend a single constitutive equation as the equation for use in the cases described in (i)-(iii). For this reason, many non-Newtonian models or constitutive equations have been proposed and most of them are empirical or semi-empirical. For more general three dimensional representation, the method of continuum mechanics is needed [1]. Although many constitutive equations have been suggested, many questions are still unsolved. Some of the continuum models do not give satisfactory results in accordance with available experimental data. For this reason, in many practical applications, empirical or semi-empirical equations have been used.

It has been shown that for many types of problems in which the flow is slow enough in the visco-elastic sense, the results given by Oldroyd's constitutive equations will be substantially equal to those of the second or third order Rivilin-Ericksen constitutive equations [2]. Thus if this is the sense in which the solutions to which problems are to be interpreted, it would seem reasonable to use the second or third order constitutive equations in carrying out the calculations. This is particularly so in view of the fact that, the calculation will generally be still simpler. For this reason, in this paper, the second order fluid model is used. The constitutive equation for the fluids of second grade (or second order fluids) is a linear relationship between the stress, the first Rivlin-Ericksen tensor, its square and the second Rivlin-Ericksen tensor. The constitutive equation has three coefficients. There are some restrictions on these coefficients due to the Clausius-Duhem inequality and the assumption that the Helmholtz free energy is a minimum in equilibrium. A comprehensive discussion on the restrictions for these coefficients has been given in [3], [4]. One of these coefficients represents the viscosity coefficient in a way similar to that of a Newtonian fluid in the absence of the other two coefficients. The restrictions on these two coefficients have not been confirmed by experiments and the sign of these material moduli is the subject of much controversy [5]. The equation of the motion of incompressible second grade fluids, in general, is of higher order than the Navier-Stokes equation. The Navier-Stokes equation is second order partial differential equation, but the equation of motion of a second order fluid is a third order partial differential equation. A marked difference between the case of the Navier-Stokes theory and that for fluids of second grade is that ignoring the nonlinearity in the Navier-Stokes equation does not lower the order of the equation however, ignoring the higher order nonlinearities in the case of the second grade fluid, reduces the order of the equation. Exact solutions are very important for many reasons. They provide a standard for checking the accuracies of many approximate methods such as numerical and empirical. Although computer techniques make the complete numerical integration of the non-linear equations feasible, the accuracy of the results can be established by a comparison with an exact solution. Many attempts to collect the exact solution of the nonlinear equations for unsteady flow of second grade fluid have been by different researcher for different geometries.

In view of several industrial and technological importance,[6] studied the problem of the exact solutions of two dimensional flows of a second order incompressible fluid by considering the rigid boundaries. Later, a linear analysis of the compressible boundary layer flow over a wall was presented by [7]. Subsequently, [8] studied the problem of Rayleigh for wavy wall while [9] examined the effect of small amplitude wall waviness upon the stability of the laminar boundary layer. Further, the problem of free convective heat transfer in a viscous incompressible fluid confined between vertical wavy wall and a particle flat wall was examined by [10], [11]. Later, [12] studied the free convective flow of a viscous incompressible fluid in porous medium between two long vertical wavy walls. Subsequently, [13] had examined the problem of MHD flow with slip effects and temperature dependent heat source in a viscous incompressible fluid confined between a long vertical wall and a parallel flat plate. Later, [14] examined the problem of elastico-viscous fluid of second order type where the bounding surface is porous and subjected to sinusoidal disturbances. Subsequently, [21], [22] studied the unsteady poiseuille flow of second order fluid in a tube of elliptical cross section and uniform cross section. [15] studied elliptical cross section on the porous boundary. Later, [23] had examined the problem of unsteady flow of an incompressible viscous electrically conducting fluid in the 
tube of elliptical cross section under the influence of the magnetic field. Subsequently, [24] studied the unsteady flow of an incompressible viscous fluid in a tube of spherical cross section on a porous boundary. Recently, [25], [26] had examined the problem of unsteady MHD flow of elasticoviscous incompressible fluid through a porous media between two parallel plates and spherical cross section under the influence of magnetic field.

In all above investigations, the fluid under consideration was viscous incompressible fluid and one of the bounding surfaces has a wavy character or bounding surface subjected to sinusoidal disturbances. In all of the above situations, not much of attention has been paid on the study of unsteady flow of second order fluid in an infinitely long tube of circular, elliptical or spherical cross section on the porous boundary. Therefore, an attempt has been made to study the effects of the transverse magnetic field on the flow of incompressible viscous electrically conducting fluid of second order type creating forced oscillations on the porous boundary. Hence the present investigation is extended work of [27], the mathematical solution is obtained for skin friction, mass flow rate and traced the paths of the fluid particles. In this aspect is also studied and during the course of discussion both nonmagnetic and magnetic cases are compared. The results are expressed in terms of a non-dimensional porosity parameter, which depends on the non-Newtonian coefficient. It is noticed that the flow properties are identical with those in the Newtonian case $(\beta=0, K \rightarrow \infty$ and $m \rightarrow 0)$.

\section{Mathematical Formulation of the Problem}

In the sense of Noll [16], a simple material is a substance for which stress can be determined with entire knowledge of the history of the strain. This is called simple fluid, if it has property that at all local states, with the same mass density, are intrinsically equal in response, with all observable differences in response being due to definite differences in the history. For any given history $g(s)$, a retarded history $g_{\psi}(s)$ can be defined as:

$$
g_{\psi}(s)=g(\psi s): 0 \leq s \leq \infty . \quad 0 \leq \psi \leq 1
$$

$\psi$ being termed as a retardation factor. Assuming that the stress is more sensitive to recent deformation than to the deformations at distant past, it has been established by Coleman and Noll [17] that the theory of simple fluids yields the theory of perfect fluids as $\psi \rightarrow 0$ and that of Newtonian Fluids as a correction (up to the order of $\psi$ ) to the theory of the perfect fluids. Neglecting all the terms of the order higher than two in $\psi$, we have incompressible elastico viscous fluid of second order type whose constitutive relation is governed by:

$$
S=-P I+\varphi_{1} E^{(1)}+\varphi_{2} E^{(2)}+\varphi_{3} E^{(1)^{2}}
$$

where

$$
E_{i j}^{1}=U_{i, j}+U_{j, i}
$$

and

$$
E_{i j}^{2}=A_{i, j}+A_{j, i}+2 U_{m, i} U_{m, j}
$$

In the above equations, $\mathrm{S}$ is the stress-tensor, $U_{i}$ and $A_{i}$ are the components of velocity and acceleration in the direction of the $i^{\text {th }}$ coordinate $X_{i}$ while $P$ is indeterminate hydrostatic pressure. The coefficients $\varphi_{1}, \varphi_{2}$ and $\varphi_{3}$ are material constants. The constitutive relation for general RivlinEricksen [18] fluid also reduces to eqn (2) when the squares and higher orders of $E^{2}$ are neglected, with the coefficients being constants. Also, the non-Newtonian models considered by Reiner [19] could be obtained from eqn (2) when $\varphi_{2}=0$ and naming $\varphi_{3}$ as the coefficient of cross viscosity. With reference to the Rivlin-Ericksen fluids, $\varphi_{2}$ may be called as the coefficient of elastico viscosity.

It has been reported that a solution of poly-iso-butylene in cetane behaves as a second order fluid. In many of the chemical processing industries, slurry adheres to the reactor vessels and gets consolidated. As a result of this, the chemical compounds within the reactor vessel percolates through the boundaries causing loss of production and consuming more reaction time. In view of such technological and industrial importance wherein the heat and mass transfer takes place in the chemical industry, the problem of considering the permeability of the bounding surfaces in the reactors attracts the attention of several investigators.

The aim of the present paper is to study a class of exact solutions for the flow of incompressible fluid of second order type by taking into account the porosity factor of the bounding surfaces and comparing the results with those in the Newtonian case. We study the disturbance due to forced oscillations of a liquid of finite depth bounded by a porous bottom. The results are expressed in terms of a non-dimensional porosity parameter $\mathrm{K}$, which depends on the non-Newtonian coefficient $\varphi_{2}$ and the frequency of excitation $\sigma$. It is noticed that the flow properties are identical with those in the Newtonian case $(\mathrm{K}=\infty)$.

If $V\left(U_{1}, U_{2}, U_{3}\right)$ is the velocity component and $F$ $\left(F_{x}, F_{y}, F_{z}\right)$ are the body forces acting on the system, then the equation of motion in $\mathrm{X}, \mathrm{Y}$ and $\mathrm{Z}$ directions are given by

$$
\begin{gathered}
\rho \frac{D U_{1}}{D T}=\rho F_{X}+\frac{\partial S_{X X}}{\partial X}+\frac{\partial S_{X Y}}{\partial Y}+\frac{\partial S_{X Z}}{\partial Z} \\
\rho \frac{D U_{2}}{D T}=\rho F_{Y}+\frac{\partial S_{Y X}}{\partial X}+\frac{\partial S_{Y Y}}{\partial Y}+\frac{\partial S_{Y Z}}{\partial Z} \\
\rho \frac{D U_{3}}{D T}=\rho F_{Z}+\frac{\partial S_{Z X}}{\partial X}+\frac{\partial S_{Z Y}}{\partial Y}+\frac{\partial S_{Z Z}}{\partial Z}
\end{gathered}
$$

where $\frac{D}{D T}=\frac{\partial V}{\partial T}+V . \nabla V$ 
If the bounding surface is porous, then the rate of percolation of the fluid is directly proportional to the cross sectional area of the filter bed and the total force, say the sum of the pressure gradient and the gravity force. In the sense of Darcy [20]

$$
q=C A\left(\frac{P_{1}-P_{2}}{H_{1}-H_{2}}+\rho G\right)
$$

where $A$ is the cross sectional area of the filter bed, $G$ is the gravitational force and $C=\frac{k}{\mu}$ in which ${ }_{k}$ is the permeability of the material and $\mu$ is the coefficient of viscosity and $q$ is the flux of the fluid. Since this law is empirical, therefore to generalize this result we must have the relation for variable thickness of the porous material. A straight forward generalization of the eqn (8) yields

$$
V=-\frac{k}{\mu}[\nabla P+\rho G \eta]
$$

where $V$ is the velocity vector and $\eta$ is the unit vector along the gravitational force taken in the-ve direction. If any other external forces are acting on the system, instead of gravitational force, then we have

$$
V=-\frac{k}{\mu}[\nabla P-\rho F]
$$

In the absence of external forces, $V=-\frac{k}{\mu} \nabla P$ which gives $\nabla P=-\frac{\mu}{k} V$

Therefore, the net resulting equation (in the dimensional form) of motions in the $\mathrm{X}, \mathrm{Y}$ and $\mathrm{Z}$ directions are

$$
\begin{aligned}
& \rho \frac{D U_{1}}{D T}=\rho F_{X}+\frac{\partial S_{X X}}{\partial X}+\frac{\partial S_{X Y}}{\partial Y}+\frac{\partial S_{X Z}}{\partial Z}-\frac{\mu}{k} U_{1} \\
& \rho \frac{D U_{2}}{D T}=\rho F_{Y}+\frac{\partial S_{Y X}}{\partial X}+\frac{\partial S_{Y Y}}{\partial Y}+\frac{\partial S_{Y Z}}{\partial Z}-\frac{\mu}{k} U_{2} \\
& \rho \frac{D U_{3}}{D T}=\rho F_{Z}+\frac{\partial S_{Z X}}{\partial X}+\frac{\partial S_{Z Y}}{\partial Y}+\frac{\partial S_{Z Z}}{\partial Z}-\frac{\mu}{k} U_{3}
\end{aligned}
$$

Introducing the following non dimensional variables as:

$$
\begin{gathered}
U_{i}=\frac{\varphi_{1} u_{i}}{\rho L} \quad T=\frac{\rho L^{2} t}{\varphi_{1}} \varphi_{2}=\rho L^{2} \beta \quad P=\frac{\varphi_{1}^{2} p}{\rho L^{2}} \\
\frac{X_{i}}{L}=x_{i} \frac{Y_{i}}{L}=y_{i} \quad \varphi_{3}=\rho L^{2} v_{c} \quad A_{i}=\frac{\varphi_{1}^{2} a_{i}}{\rho^{2} L^{3}} \\
S_{i, j}=\frac{\varphi_{1}^{2} s_{i, j}}{\rho L^{2}} E_{i, j}^{(1)}=\frac{\varphi_{1} e_{i, j}^{(1)}}{\rho L^{2}} E_{i, j}^{(2)}=\frac{\varphi_{1}^{2} e_{i, j}^{(2)}}{\rho^{2} L^{4}} \quad k=\frac{L^{2} K}{\rho}
\end{gathered}
$$

$$
F_{i}=\frac{\varphi_{1}^{2}}{\rho^{2} L^{3}} f_{i} \quad \mu=\frac{\varphi_{1}}{\rho} \quad V=\frac{\varphi_{1} v}{\rho L} \quad M=\frac{\varphi_{1} m}{L^{2}}
$$

where $T$ is the (dimensional) time variable, and $\rho$ the mass density and $L$ a characteristic length.

The non dimensional form of Eq (10) will now be:

$$
v=-K(\nabla p-f)
$$

In the absence of external forces $v=-K \nabla p$ which yields

$$
\nabla p=-\frac{v}{K}
$$

We consider a class of plane flows given by the velocity components

$$
u_{1}=u(y, t) \text { and } u_{2}=0
$$

in the directions of rectangular Cartesian coordinates $\mathrm{x}$ and $\mathrm{y}$. The velocity field given by eq.(16) identically satisfies the incompressibility condition. The stress can now be obtained in a non-dimensional form as:

$$
\begin{gathered}
s_{x x}=-p+v_{c}\left(\frac{\partial u}{\partial y}\right)^{2} \\
s_{y y}=-p+\left(v_{c}+2 \beta\right)\left(\frac{\partial u}{\partial y}\right)^{2} \\
s_{x y}=\frac{\partial u}{\partial y}+\beta \frac{\partial}{\partial y}\left(\frac{\partial u}{\partial t}\right)
\end{gathered}
$$

In view of the above, the equation of motion in the $x$-direction is given by:

$$
\frac{\partial u}{\partial t}=-\frac{\partial p}{\partial x}+\frac{\partial^{2} u}{\partial y^{2}}+\beta \frac{\partial}{\partial t}\left(\frac{\partial^{2} u}{\partial y^{2}}\right)-\left(\frac{1}{K}+m\right) u+f_{x}
$$

where $f_{x}$ is the external force acting along the x-direction.

The equation of motion in the $y$-direction in the absence of any external forces is given by:

$$
0=-\frac{\partial p}{\partial y}+\left(2 \beta+v_{c}\right) \frac{\partial}{\partial y}\left(\frac{\partial u}{\partial y}\right)^{2}
$$

The pressure gradient in eqn (20) can only be a function of time for this flow.

Using Eq. (20) if

$$
\begin{gathered}
-\frac{\partial p}{\partial x}=\xi(t) \\
\int_{p_{0}(t)}^{p} \partial p=-\xi(t) \int_{0}^{x} \partial x
\end{gathered}
$$




$$
p=p_{0}(t)-\xi(t) x
$$

where $p_{0}(t)$ is the initial pressure.

From Eq (21)

$$
\frac{\partial p}{\partial y}=\left(2 \beta+v_{c}\right) \frac{\partial}{\partial y}\left(\frac{\partial u}{\partial y}\right)^{2}
$$

which on integration w.r.t y yields:

$$
p=\left(2 \beta+v_{c}\right)\left(\frac{\partial u}{\partial y}\right)^{2}+\text { a function of } x \text { say } \lambda(x)
$$

Using Eq.(22) and Eq.(23)

$$
\lambda(x)=p_{0}(t)-\xi(t)-\left(2 \beta+v_{c}\right)\left(\frac{\partial u}{\partial y}\right)^{2}
$$

Using Eq.(24) in Eq.(23)

$$
p=p_{0}(t)-\xi(t) x
$$

Considering $\xi(t)=0$, and using eq.(25) in Eq (20) the flow characterized by the velocity is given by:

$$
\frac{\partial u}{\partial t}=\frac{\partial^{2} u}{\partial y^{2}}+\beta \frac{\partial}{\partial t}\left(\frac{\partial^{2} u}{\partial y^{2}}\right)-\left(\frac{1}{K}+m\right) u+f_{x}
$$

where $\mathrm{K}$ is the non-dimensional porosity constant and $f_{x}$ is the external force (non-dimensional) acting along the $\mathrm{x}$-direction. It may be noted that the presence of $\beta$ changes the order of differential from two to three.

\section{Forced Oscillations of a Liquid of Finite Depth Bounded by Rigid Bottom}

Let the fluid of the depth $L h$, bounded by the rigid bottom $y=0$, be influenced by the (non-dimensional) external force $F e^{i \sigma t}$ in the $x$ direction. A magnetic field of constant strength is supposed to be applied parallel to y direction. The induced magnetic field is negligible as comparing with applied magnetic field, the flow is laminar it is valid for magnetic Reynolds number less than unity

In such a situation eqn (26) will now get modified as:

$$
\frac{\partial u}{\partial t}=\frac{\partial^{2} u}{\partial y^{2}}+\beta \frac{\partial}{\partial t}\left(\frac{\partial^{2} u}{\partial y^{2}}\right)-\left(\frac{1}{K}+m\right) u+F e^{i \sigma t}
$$

with the no-slip condition at the bottom

$$
u(0, t)=0
$$

and the free surface condition on the top

$$
s_{x y}=0 \text {, at } y=h
$$

Assuming the trial solution as:

$$
\begin{gathered}
u(y, t)=F f(y) e^{i \sigma t} \\
f^{\prime \prime}(y)-p^{2} f(y)=-\frac{1}{(1+i \beta \sigma)}
\end{gathered}
$$

Where

$$
p^{2}=\frac{i \sigma+\left(\frac{1}{K}+m\right)}{1+i \beta \sigma}=\frac{\left(\beta \sigma^{2}+\left(\frac{1}{K}+m\right)+i\left(\sigma-\beta \sigma\left(\frac{1}{K}+m\right)\right)\right.}{\left(1+\beta^{2} \sigma^{2}\right)}
$$

When expressed in polar form

$$
p=r\left(\cos \left(\frac{\pi}{4}-\frac{\varepsilon}{2}\right)+i \sin \left(\frac{\pi}{4}-\frac{\varepsilon}{2}\right)\right)
$$

where,

$$
\begin{gathered}
r=\frac{\left[\left(\beta \sigma^{2}+\left(\frac{1}{K}+m\right)\right)^{2}+\left(\sigma-\beta \sigma\left(\frac{1}{K}+m\right)\right]^{1 / 4}\right.}{\sqrt{\left(1+\beta^{2} \sigma^{2}\right)}}, \\
\varepsilon=\tan ^{-1}(Q) \text { and } Q=\frac{\left(\frac{1}{K}+m\right)+\beta \sigma^{2}}{\sigma-\beta \sigma\left(\frac{1}{K}+m\right)}
\end{gathered}
$$

Also the conditions satisfied by $f(y)$ are

$$
f(0)=0, f^{\prime}(h)=0
$$

This yields the solution:

$$
f(y)=\frac{1}{p^{2}(1+i \beta \sigma)}\left[1-\frac{\cosh p(h-y)}{\cosh p h}\right]
$$

In view of eqn (30)

$$
u(y, t)=R P \frac{F e^{i \sigma t}}{p^{2}(1+i \beta \sigma)}\left[1-\frac{\cosh p(h-y)}{\cosh p h}\right]
$$

hence,

$$
u(y, t)=\frac{F[A-B]}{\left[\left(\frac{1}{K}+m\right)^{2}+\sigma^{2}\right][\cosh (2 a h)+\cos (2 b h)]}
$$

Where

$$
A=\left(\begin{array}{c}
\left(\cos \sigma t\left(\frac{1}{K}+m\right)+\sigma \sin \sigma t\right)\{\cosh 2 a h+\cos 2 b h- \\
\cosh a(2 h-y) \cos b y-(\cosh a y) \cos b(2 h-y)\}
\end{array}\right)
$$

$$
B=\left(\begin{array}{l}
\left(\sin \sigma t\left(\frac{1}{K}+m\right)-\sigma \cos \sigma t\right)\{\sinh a(2 h-y) \\
\sin b y+\sinh a y \sin b(2 h-y)\}
\end{array}\right)
$$


on $y=h$, the velocity is:

$$
u(h, t)=\frac{F\left[A_{1}-B_{1}\right]}{\left[\left(\frac{1}{K}+m\right)^{2}+\sigma^{2}\right][\cosh (2 a h)+\cos (2 b h)]}
$$

where

$$
\begin{gathered}
A_{1}=\left(\begin{array}{l}
\left(\cos \sigma t\left(\frac{1}{K}+m\right)+\sigma \sin \sigma t\right)\{\cosh 2 a h \\
+\cos 2 b h-2 \cosh a h \cos b h\}
\end{array}\right) \\
B_{1}=\left(\left(\sin \sigma t\left(\frac{1}{K}+m\right)-\sigma \cos \sigma t\right)\{2 \sinh a h \sin b h\}\right)
\end{gathered}
$$

The paths of particles may be obtained by integrating eqn (36) with respect to $t$

$$
x=R P \frac{F e^{i \sigma t}}{i \sigma p^{2}(1+i \beta \sigma)}\left[1-\frac{\cosh p(h-y)}{\cosh p h}\right]
$$

the constant of integration may be conveniently taken to be zero for particles starting from the same point (taken as origin ) on the bottom

For the case of large $h$,

$$
u=\frac{F}{\left(\frac{1}{K}+m\right)^{2}+\sigma^{2}}\left[\begin{array}{l}
\left(\cos \sigma t\left(\frac{1}{K}+m\right)+\sigma \sin \sigma t\right) \\
+e^{-y r \cos \left(\frac{\pi}{4}-\frac{\varepsilon}{2}\right)} \cdot \sin \left\{y r \sin \left(\frac{\pi}{4}-\frac{\varepsilon}{2}\right)-\sigma t\right\}
\end{array}\right]
$$

which for $y>>\delta$, reduces to

$$
u \approx \frac{F}{\left(\frac{1}{K}+m\right)^{2}+\sigma^{2}}\left(\cos \sigma t\left(\frac{1}{K}+m\right)+\sigma \sin \sigma t\right)
$$

where $\delta=\frac{1}{r}$

The paths of the particles in this case are given by

$$
x=\frac{F}{\left(\frac{1}{K}+m\right)^{2}+\sigma^{2}}\left[\begin{array}{c}
\left(\sin \sigma t\left(\frac{1}{K}+m\right) \frac{1}{\sigma}-\sin \sigma t\right) \\
+e^{-y r \cos \left(\frac{\pi}{4}-\frac{\varepsilon}{2}\right)} \cdot \cos \left\{y r \cos \left(\frac{\pi}{4}-\frac{\varepsilon}{2}\right)-\sigma t\right\}
\end{array}\right]
$$

which for $y>>$,

$$
x \approx \frac{F}{\left(\frac{1}{K}+m\right)^{2}+\sigma^{2}}\left(\frac{\sin \sigma t}{\sigma}\left(\frac{1}{K}+m\right)-\cos \sigma t\right)
$$

$$
x^{*}=\frac{R P x\left(\left(\frac{1}{K}+m\right)^{2}+\sigma^{2}\right)}{F\left(\frac{\sin \sigma t}{\sigma}\left(\frac{1}{K}+m\right)-\cos \sigma t\right)}
$$

$y^{*}=\frac{y\left(1+\beta^{2} \sigma^{2}\right)^{\frac{1}{4}}}{\sqrt{2}}\left[\frac{\left(\frac{1}{K}+M+\beta \sigma^{2}\right)^{2}+\left(\sigma-\beta \sigma\left(\frac{1}{K}+m\right)^{2}\right.}{\left(1+\beta^{2} \sigma^{2}\right)^{2}}\right]^{\frac{1}{4}}$

Phase parameter in this case is given by:

$$
\phi=\frac{\varepsilon}{\sigma}=\frac{\tan ^{-1}(Q)}{\sigma}
$$

The skin-frication is given by:

Skin-frication $\frac{\partial u}{\partial y}+\beta\left[\frac{\partial^{2} u}{\partial y \partial t}-\frac{\partial^{2} u}{\partial y^{2}}\right]$ at $y=0$.

The mass flow rate is given by integrating the equation (36).

Mass flow rate $=\int_{0}^{1} u(y, t) d y$

\section{Conclusion}

In this paper obtained expression for skin friction and mass flow rate along with paths of the fluid particles. As $K \rightarrow \infty$, $m \rightarrow 0$ the results obtained for the velocity field, paths of the particles are in agreement with those of Pattabhi Ramacharyulu [6]. In the absence of external forces, the results coincide with that of Ramana Murthy and Kulkarni [14]. The case of Newtonian fluid can be realized as $\beta \rightarrow 0$ $K \rightarrow 0 \& m \rightarrow 0$.

\section{References}

[1] K. R. Rajagopal, P. L. Koloni, "Continuum Mechanics and its Applications”, Hemisphere Press, Washington, DC, 1989.

[2] K. Walters, "Relation between Coleman-Nall, Rivlin-Ericksen, Green-Rivlin and Oldroyd fluids", ZAMP, 21, 1970 pp. 592-600.

[3] J. E. Dunn, R. L. Fosdick, "Thermodynamics stability and boundedness of fluids of complexity 2 and fluids of second grade”, Arch. Ratl. Mech. Anal, 56, 1974, pp. 191-252.

[4] J. E. Dunn, K. R. Rajagopal, "Fluids of differential type-critical review and thermodynamic analysis", J. Eng. Sci., 33, 1995, pp. 689-729.

[5] K. R. Rajagopal, "Flow of visco-elastic fluids between rotating discs”, Theor. Comput. Fluid Dyn., 3, 1992, pp. 185-206.

[6] N. Ch. Pattabhi Ramacharyulu, "Exact solutions of two dimensional flows of second order fluid", App. Sc Res, Sec-A, 15. 1964, pp. 41-50. 
[7] S. G. Lekoudis, A. H. Nayef and Saric., "Compressible boundary layers over wavy walls", Physics of fluids, 19, 1976, pp. 514-19.

[8] P. N. Shankar, U. N. Shina, "The Rayeigh problem for wavy wall”, J. Fluid Mech, 77, 1976, pp. 243-256.

[9] M. Lessen, S. T. Gangwani, "Effects of small amplitude wall waviness upon the stability of the laminar boundary layer", Physics of the fluids, 19, 1976, pp. 510-513.

[10] K. Vajravelu, K. S. Shastri, "Free convective heat transfer in a viscous incompressible fluid confined between a long vertical wavy wall and a parallel flat plate", J. Fluid Mech, 86, 1978, pp. 365-383.

[11] U. N. Das, N. Ahmed, "Free convective MHD flow and heat transfer in a viscous incompressible fluid confined between a long vertical wavy wall and a parallel flat wall”, I. J. Pure \& App. Math, 23, 1992, pp. 295-304.

[12] R. P Patidar, G. N. Purohit, "Free convection flow of a viscous incompressible fluid in a porous medium between two long vertical wavy walls", I. J. Math, 40, 1998, pp. 76-86.

[13] R. Taneja, N. C. Jain, "MHD flow with slip effects and temperature dependent heat source in a viscous in compressible fluid confined between a long vertical wavy wall and a parallel flat wall”, J. Def. Sci., 2004, pp. 21-29.

[14] Ch. V. R. Murthy, S. B. Kulkarni, "On the class of exact solutions of an incompressible fluid flow of second order type by creating sinusoidal disturbances", J. Def. Sci, 57, 2, 2007, pp. 197-209.

[15] S. B. Kulkarni, "Unsteady poiseuille flow of second order fluid in a tube of elliptical cross section on the porous boundary", Special Topics \& Reviews in Porous Media., 5, 2014, pp. 269276.

[16] W. Noll, "A mathematical theory of mechanical behaviour of continuous media", Arch. Ratl. Mech. \& Anal., 2, 1958, pp. 197-226.

[17] B. D. Coleman, W. Noll, "An approximate theorem for the functionals with application in continuum mechanics", Arch. Ratl. Mech and Anal, 6, 1960, pp. 355-376.
[18] R. S. Rivlin, J. L. Ericksen, "Stress relaxation for isotropic materials", J. Rat. Mech, and Anal, 4, 1955, pp. 350-362.

[19] M. Reiner, "A mathematical theory of diletancy", Amer. J. ofMaths, 64, 1964, pp. 350-362.

[20] H. Darcy, "Les FontainesPubliques de la Ville de, Dijon, Dalmont, Paris" 1856.

[21] E. M. Erdogan, E. Imrak, "Effects of the side walls on the unsteady flow of a Second-grade fluid in a duct of uniform cross-section", Int. Journal of Non-Linear Mechanics, 39, 2004, pp. 1379-1384.

[22] S. Islam, Z. Bano, T. Haroon and A. M. Siddiqui, "Unsteady poiseuille flow of second grade fluid in a tube of elliptical cross-section", 12, 4, 2011. 291-295.

[23] S. B. Kulkarni, "Unsteady flow of an incompressible viscous electrically conducting fluid in tub of elliptical cross section under the influence of magnetic field", International Journal of Mathematical, Computational, Physical and Quantum Engineering, 8 (10), 2014, pp. 1311-1317.

[24] S. B. Kulkarni, "Unsteady poiseuille flow of an incompressible viscous fluid in a tube of spherical cross section on a porous boundary", International Journal of Mechanical Aero Space Industrial and Mechatronics Engineering, 9 (2), 2015, pp. 240 246.

[25] S. B. Kulkarni, "Unsteady MHD flow of elastico-viscous incompressible fluid through a porous media between two parallel plates under the influence of magnetic field", Defence Science Journal, 65 (2), 2015, pp. 119-125.

[26] S. B. Kulkarni, "Unsteady MHD flow of elastic-viscous fluid in tube of spherical cross section on porous boundary", International Journal of Mechanical Aero Space Industrial and Mechatronics Engineering, 9 (4), 2015, pp. 590-596.

[27] S. B. Kulkarni, G. G. Bhide and G. S. Kulkarni, "On the class of exact solution of MHD fluid flow of second order type by creating forced oscillation on the porous boundary", International Journal of Recent Advances in Engineering and Technplogy, 4 (8), 2016, pp, 1-8. 